\title{
Intelligent Control for Formation Flight of UAVs Based on ANFIS
}

\author{
Amr Sarhan ${ }^{1, a *}$, Shiyin Qin ${ }^{2, b}$ \\ ${ }^{1}$ School of Automation Science and Electrical Engineering Beihang University, China \\ ${ }^{2}$ School of Automation Science and Electrical Engineering Beihang University, China \\ aamrsarhan_39@hotmail.com, ${ }^{\text {b }}$ qsy@buaa.edu.cn
}

Keywords: Control, Intelligent Control, ANFIS, Formation Control, UAVs.

\begin{abstract}
In this paper, a leader-follower formation flight controller based on ANFIS is developed. The simulation results present the validity and good performance of the developed formation controller in several flight conditions; straight-path trajectory, circular-path trajectory, spiral-path trajectory, and finally obstacle collision path trajectory.
\end{abstract}

\section{Introduction}

Recently, multi-UAV architectures have been given important interests due to their several applications in civilian and military missions [1]. Multi-UAV architecture has the ability to execute complex missions with lower cost and higher efficiency [2,3]. All missions for single UAV can be executed more efficiently by multi-UAVs architectures [4]. Multi-UAV architectures can be classified according to the coupling between the UAVs to physical coupling, swarms, intention cooperation, and finally formation. In physical coupling, the UAVs are connected by physical links and the UAVs motion is restricted by the motion of each UAV member [5,6]. In swarms, the motion of all the UAV members is without any predefined spatial patterns and doesn't cause formation. In intention cooperation, the architecture moves according to paths specified by individual missions that should be allocated to execute the overall mission. While in formation, the focus of this study, UAVs members are constrained to keep the formation without any physical coupling.

Formation flight has many merits such as increasing the duration of the flight by reducing the drag and save the energy [7], extending covering the area of the sensors, and finally increased the system redundancy $[8,9]$. The formation control problem contains two essential issues; the construction of the formation and the preserving the formation [10]. In the existing literature, the formation approach is classified to leader-follower strategy, virtual structure strategy, and behavior-based strategy [11].

The leader-follower strategy is most attractive because of its simplicity and its ability to adjust the configuration size according to the new conditions. Each follower UAV in the formation has at least one leader which track it with some offset. The merits of the leader-follower strategy are simplifying the problems of the formation control to individual tracking problems and it is easy to analyze and implement [12]. There are two modes: leader mode and front mode. In the leader mode, each follower takes the data from the leader while in front mode takes the data from prior one. Various control approaches have been proposed to control the leader-follower formation flight. Some of these approaches are $H_{\infty}$ [13], model predictive [14,15], backstepping [10], sliding mode [16], fuzzy logic [17], and State-Dependent Riccati Equation control [18].

In this paper, an adaptive neuro-fuzzy inference system (ANFIS) is utilized to control the leader-follower formation flight. ANFIS approach is utilized to design the guidance outer loop in addition to control the UAV dynamics in the inner loop. Three fuzzy logic modules are designed to maintain the desirable separation of the follower UAV with the leader UAV.

\section{Problem Formulation}

UAV Nonlinear Dynamic Model. The dynamics of the UAV in motion can be given by Newton's $2^{\text {nd }}$ law which is suitable in the inertial frame. A complete 6-DOF non-linear Aerosonde UAV 
dynamic model [19] is demonstrated in the forthcoming discussion. The Aerosonde UAV is a system with six degrees of freedom [20], its nonlinear model is described by 12 dynamic variables: body frame velocities $(u, v, w)$, Euler angles $(\varphi, \theta, \psi)$, angular velocities $(p, q, r)$, and inertial positions $\left(p_{N}, p_{E}, h\right)$, on the other hand, the model depends on external forces $\left(f_{x}, f_{y}, f_{z}\right)$ and moments $(l, m, n)$. The dynamic model is summarized in Eq.1.

$$
\left\{\begin{aligned}
\dot{u}= & r v-q w+\frac{f_{x}}{m} . \\
\dot{v}= & p w-r u+\frac{f_{y}}{m} . \\
\dot{w}= & q u-p v+\frac{f_{z}}{m} . \\
\dot{\varphi}= & p+q \sin \varphi \tan \theta+r \cos \varphi \tan \theta . \\
\dot{\theta}= & q \cos \varphi-r \sin \varphi . \\
\dot{\psi}= & \frac{q \sin \varphi+r \cos \varphi}{\cos \theta} . \\
\dot{p}= & c_{1} q r+c_{2} p q+c_{3} l+c_{4} n . \\
\dot{q}= & c_{5} p r-c_{6}\left(p^{2}-r^{2}\right)+c_{7} m . \\
\dot{r}= & c_{8} p q-c_{2} q r+c_{4} l+c_{9} n . \\
\dot{p}_{N}= & u \cos \theta \cos \psi+v(\sin \varphi \sin \theta \cos \psi-\cos \varphi \sin \psi) \\
& \quad+w(\sin \varphi \sin \psi+\cos \varphi \sin \theta \cos \psi) . \\
\dot{p}_{E}= & u \cos \theta \sin \psi+v(\cos \varphi \cos \psi+\sin \varphi \sin \theta \sin \psi) \\
& +w(\cos \varphi \sin \theta \sin \psi-\sin \varphi \cos \psi) . \\
\dot{h}= & u \sin \theta-v \sin \varphi \cos \theta-w \cos \varphi \cos \theta .
\end{aligned}\right.
$$

where $c_{1}, \ldots, c_{9}$ are functions of moments of inertia $\left(J_{x}, J_{y}, J_{z}\right) .\left(f_{x}, f_{y}, f_{z}\right)$ and $(l, m, n)$ are generally due to gravity, aerodynamics, and propulsion. These variables rely on mass $(m)$, gravity $(g)$, Euler angles, air density $(\rho)$, airspeed, surface area of the wing $(S)$, angular velocities, angle of attack $(\alpha)$, side slip angle $(\beta)$, control surface configuration $\left(\delta_{a}, \delta_{e}, \delta_{r}\right)$, engine acceleration $\left(\delta_{t}\right)$, area, aerodynamic coefficient and torque of the propeller, the efficiency of the engine, and the aerodynamic coefficients.
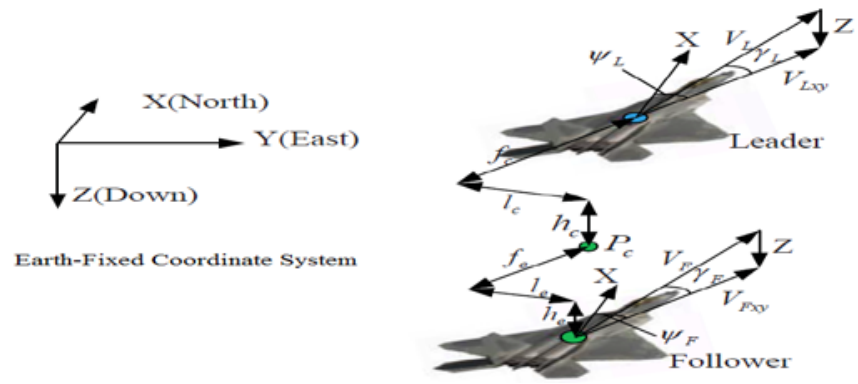

Fig. 1 3-D Leader-Follower Formation Geometry.

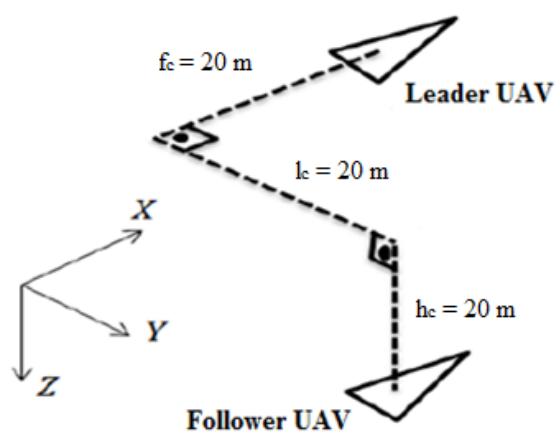

Fig. 2 Desired formation geometry.

Formation Geometry. In this work, the leader fixed body frame in Fig. 1 is chosen as a reference frame. $L$ and $F$ represent the leader and the follower UAV, respectively. The point $P_{c}$ represents the reference point where the follower UAV should be on through the formation flight. The point $P_{c}$ is located as longitudinally distance $f_{c}$, laterally distance $l_{c}$, and vertically distance $h_{c}$ from the leader UAV. The angle between the leader longitudinal axis and the north is $\psi_{L}$. The current leader position and follower position is $\left[\begin{array}{lll}x_{L} & y_{L} & z_{L}\end{array}\right]^{T}$ and $\left[\begin{array}{lll}x_{F} & y_{F} z_{F}\end{array}\right]^{T}$, respectively. The lateral distance error $l_{e}$, longitudinal distance error $f_{e}$, and vertical distance error $h_{e}$ for the follower UAV can be calculated as in Eq.2. Details of this geometry formulation can be found in [19,21]. 
$\left[\begin{array}{l}l_{e} \\ f_{e} \\ h_{e}\end{array}\right]=\left[\begin{array}{ccc}\sin \psi_{L} & -\cos \psi_{L} & 0 \\ \cos \psi_{L} & \sin \psi_{L} & 0 \\ 0 & 0 & 1\end{array}\right]\left[\begin{array}{l}x_{L}-x_{F} \\ y_{L}-y_{F} \\ z_{L}-z_{F}\end{array}\right]-\left[\begin{array}{l}l_{c} \\ f_{C} \\ h_{c}\end{array}\right]$

The leader UAV heading angle $\psi_{L}$ can be getting trigonometrically as in equation (3).

$$
\left[\cos \psi_{L} \sin \psi_{L}\right]^{T}=\left(V_{L x}^{2}+V_{L y}^{2}\right)^{-0.5}\left[V_{L x} V_{L y}\right]^{T}
$$

where $\left[V_{L x} V_{L y}\right]^{T}$ is the projections of the leader UAV velocity along the earth-fixed. For this formation problem, the first derivative of the errors will be as in equation (4).

$$
\left[\begin{array}{c}
\dot{l}_{e} \\
\dot{f}_{e} \\
\dot{h}_{e}
\end{array}\right]=\left[\begin{array}{c}
V_{F x y} \sin \left(\psi_{F}-\psi_{L}\right)+\dot{\psi}_{L}\left(f_{e}+f_{c}\right) \\
V_{L x y}-V_{F x y} \cos \left(\psi_{F}-\psi_{L}\right)-\dot{\psi}_{L}\left(l_{e}+l_{c}\right) \\
V_{L} \sin \gamma_{L}-V_{F} \sin \gamma_{F}
\end{array}\right]
$$

\section{Controller Design}

ANFIS is a combination between fuzzy logic and artificial neural network in the field of artificial intelligence [22]. Fuzzy logic is excellent at dealing with uncertainty and decision making. However, the main defect is the inability to learn [23]. On the other hand, the artificial neural network has the ability to learn but don't have the ability to make decisions. The artificial neural network aids in minimizing the error besides to optimize the controller parameters. Harvest the beneficial between two types fuzzy logic and artificial neural network, new hybrid Neuro-Fuzzy systems have come out. ANFIS is utilized to design the low-level flight controller for single UAV, in addition, to design the top-level flight controller to keep the formation.

Low-Level Controller for single UAV. For individual UAV in the formation, an autonomous flight controller is designed based on ANFIS as in Fig.3. Three fuzzy modules are designed for each UAV to achieve this objective. Two modules are designed for the longitudinal autopilot to achieve the desired altitude and airspeed. The other module is designed for the lateral autopilot to achieve the desired heading angle. In this way, each UAV in the formation is controlled in the three-dimensional space. The performance and robustness of the low-level flight controller based on ANFIS were demonstrated in details in our previous work [24].

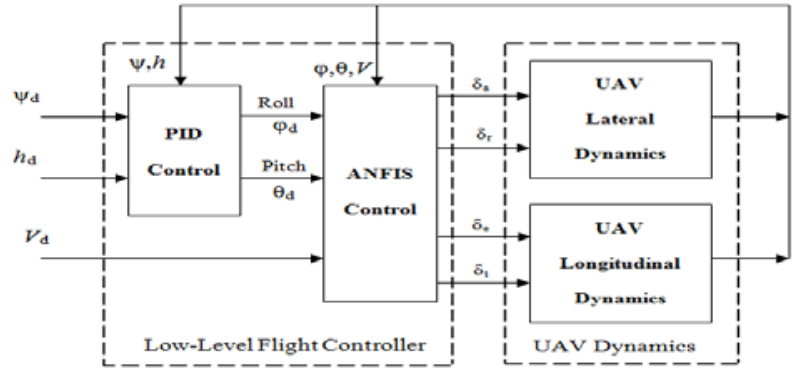

Fig. 3 Low-level flight controller.

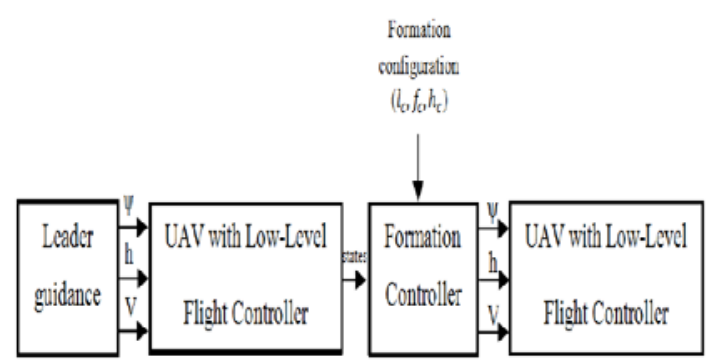

Fig. 4 Top-level flight controller.

Top-Level Flight Controller. In order to design the formation flight controller, three ANFIS modules were designed for maintaining $h_{c}=20 \mathrm{~m}, l_{c}=20 \mathrm{~m}, f_{c}=20 \mathrm{~m}$. The designed ANFIS membership functions are shown in Fig.5.

\section{Simulation results}

In this work, MATLAB ANFIS Editor Toolbox is utilized to design the inference engines. MATLAB and Aerosim software are utilized to study the performance of the designed flight control system. A nonlinear Aerosonde fixed-wing UAV model was used in the simulation. The validation simulations include several flight path trajectories as straight-path trajectory, circular-path trajectory, 
spiral-path trajectory, and finally path trajectory with obstacles. The simulation results in Fig.6-Fig.9 demonstrate that the follower UAV can successfully track the leader UAV. In addition, the tracking errors in $\mathrm{X}$-axis, Y-axis, and Z-axis tend to 0 rapidly.

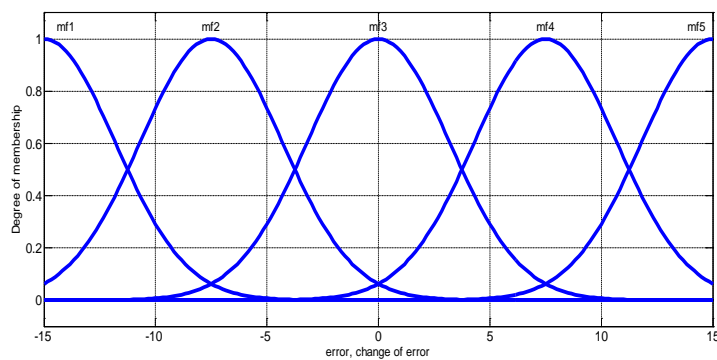

a) Error and Change of Error of Vertical Formation.

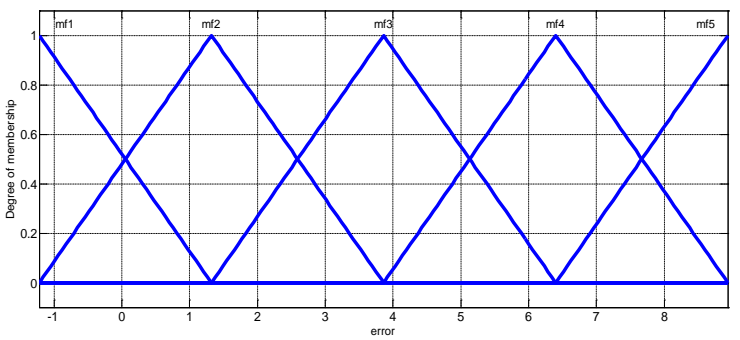

c) Error of the Lateral Formation.

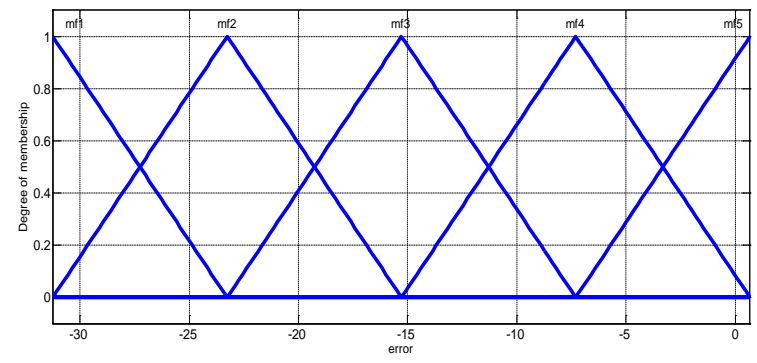

e) Error of the Longitudinal Formation.

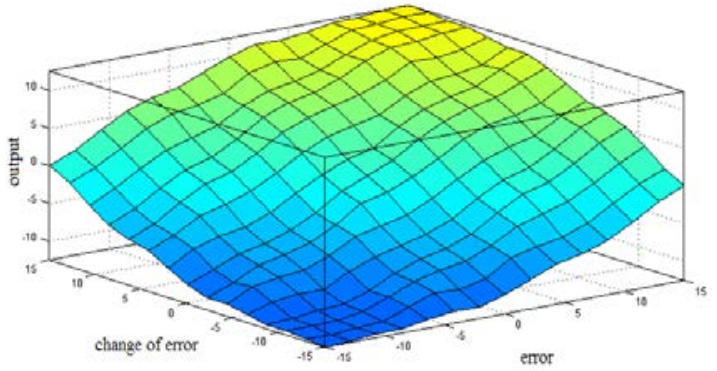

b) Output Surface of Vertical Formation.

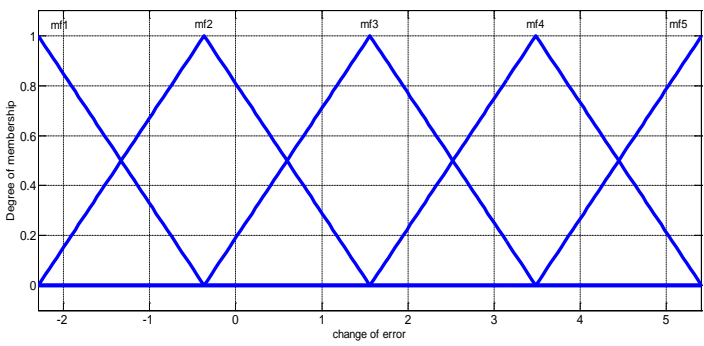

d) Change of Error of the Lateral Formation.

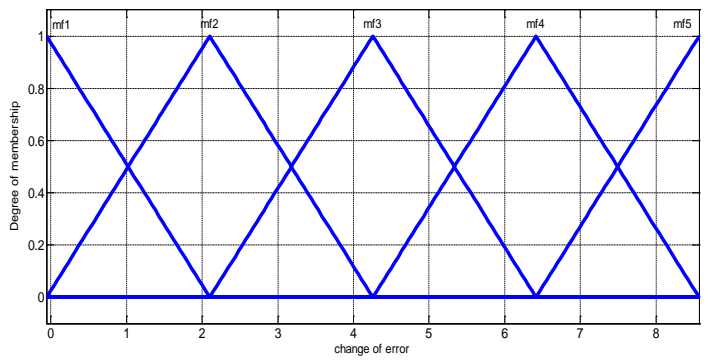

f) Change of Error of the Longitudinal Formation. Fig. 5 Membership Functions for Top-Level Flight Controller Design.
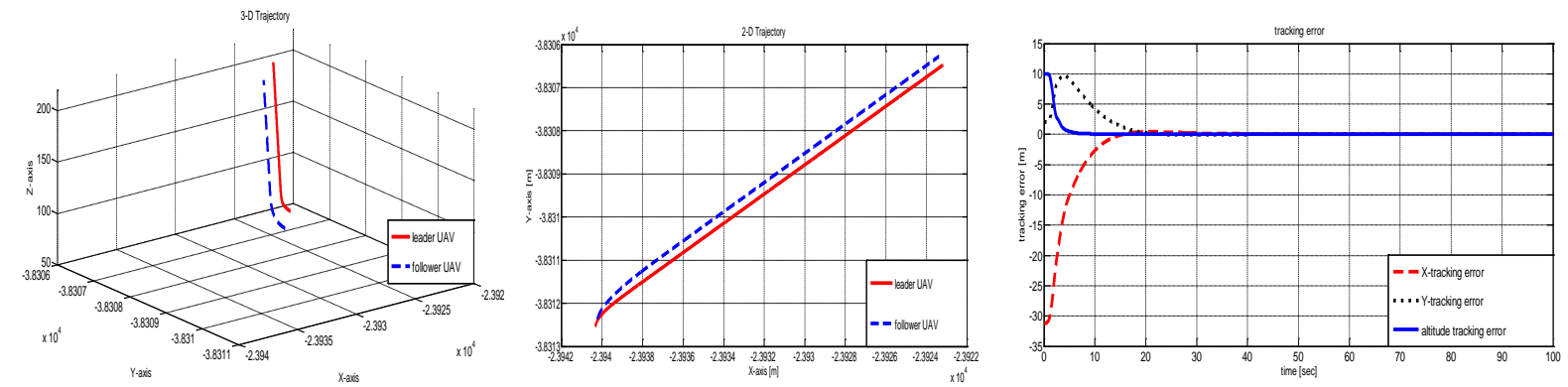

a) 3-Dimentional Trajectory. b) Top-view of the Trajectory.

Fig. 6 Straight-Path Trajectory. c) The Tracking Errors. 


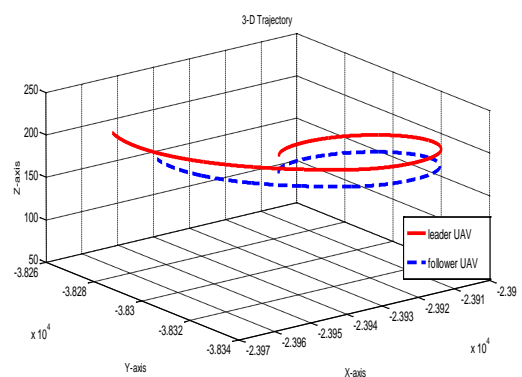

a) 3-Dimentional Trajectory.

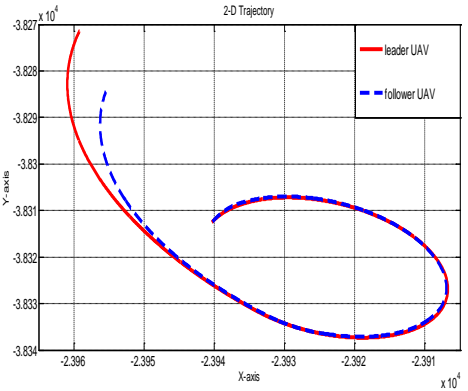

b) Top-view of the Trajectory.

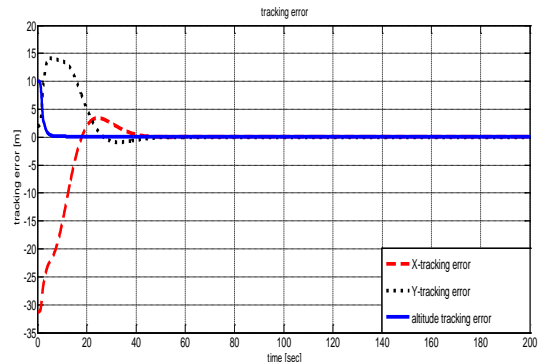

c) The Tracking Errors.

Fig. 7 Circular-Path Trajectory.

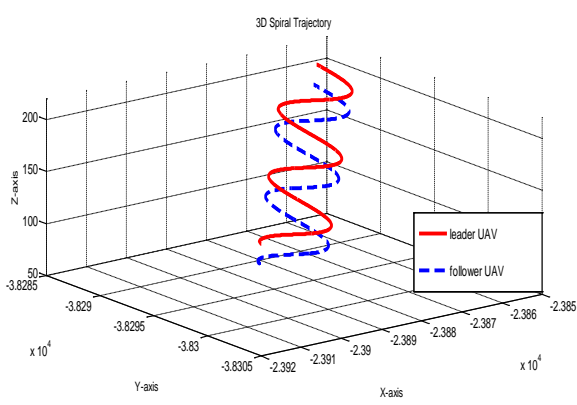

a) 3-Dimentional Trajectory.

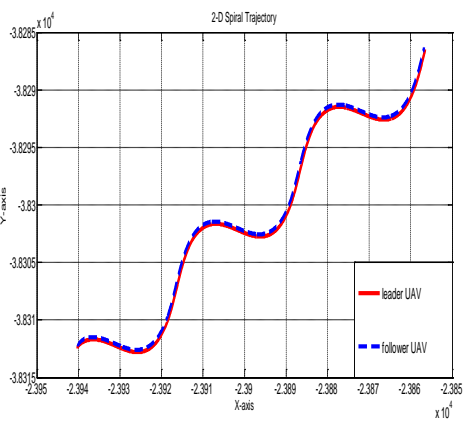

b) Top-view of the Trajectory.

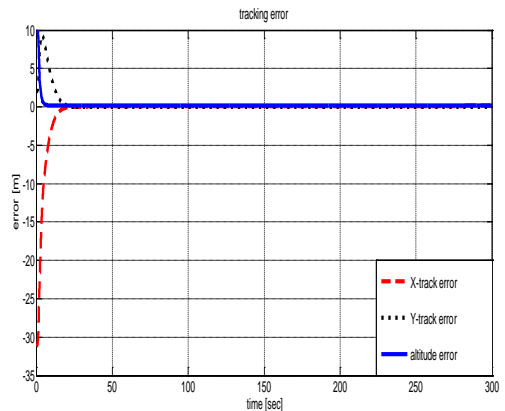

c) The Tracking Errors.

Fig. 8 Spiral-Path Trajectory.

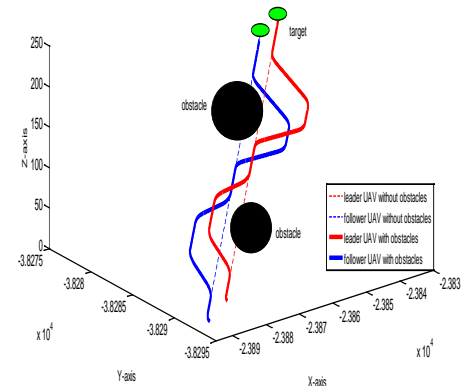

a) 3-Dimentional Trajectory.

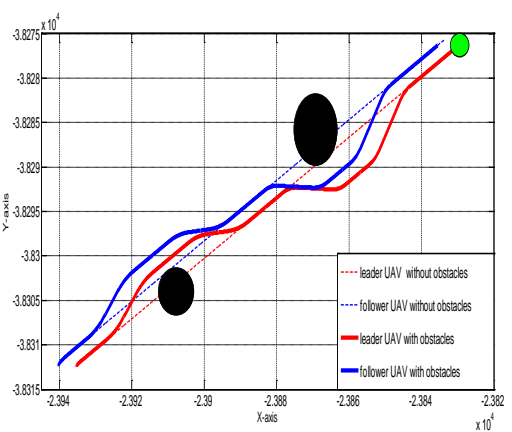

b) Top-view of the Trajectory.

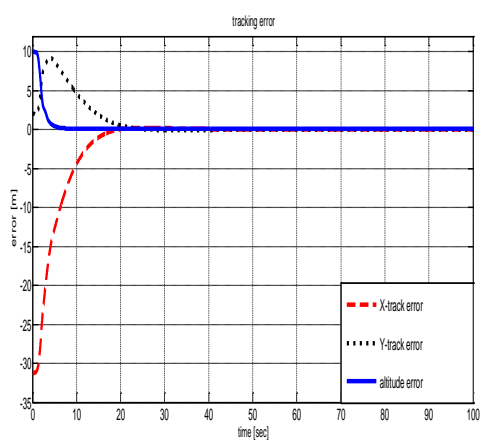

c) The Tracking Errors.

Fig. 9 Path Trajectory with Obstacles.

\section{Conclusion}

A leader-follower formation flight controller is designed based on ANFIS. The formation consists of a leader UAV and one follower UAV. The formation controller keeps the follower UAV in the predetermined position. The developed formation flight based on ANFIS is tested and evaluated in several flight conditions; straight-path trajectory, circular-path trajectory, spiral-path trajectory, and finally path trajectory with obstacles. The simulation results present the validity and good performance of the developed formation controller.

\section{Acknowledgement}

This work was partly supported by Natural Science Foundation of China under Grant U1435220.

\section{References}

[1] QIU Qi, WU Sen Tang, Robust Formation Flight Control System Design For Multi-UAVs, proceeding of the 31st Chinese control conference, Hefei, China, 2012. 
[2] Xueyuan Li, Xuejun Zhang, Huaxian Liu, and Xiangmin Guan, Formation Reconfiguration Based on Distributed Cooperative Co-evolutionary for Multi-UAV, $12^{\text {th }}$ IEEE World conference on Intelligent Control and Automation (WCICA), Guilin, China, 2016.

[3] JAMES R. RIEHL, GAEMUS E. COLLINS, JOA O P. HESPANHA, Cooperative Search by UAV Teams: A Model Predictive Approach using Dynamic Graphs, IEEE Transactions on Aerospace and Electronic Systems, Vol.47, 2011.

[4] Leszek Ambroziak, Zdzisław Gosiewski, Two stage switching control for autonomous formation flight of unmanned aerial vehicles, Aerospace Science and Technology, Vol.46, ELSEVIER, 2014.

[5] Maza, K. Kondak, M. Bernard, A. Ollero, Multi-UAV Cooperation and Control for Load Transportation and Deployment, Journal of Intelligent and Robotic Systems, Vol.57, 2010.

[6] Kimon P. Valavanis, George J. Vachtsevanos, Handbook of Unmanned Aerial Vehicles, Springer Dordrecht Heidelberg New York London, 2015.

[7] H. Weimerskirch, J. Martin, Y. Clerquin, P. Alexandre, S. Joraskova, Energy saving in flight formation, International Journal of Science, Vol. 413 697-698, 2001.

[8] Ji Xiangyu, Wu Sentang, Liu Xiang, Du Yang, Tang Jiqiang, Research and Design on Physical Multi-UAV System for Verification of Autonomous Formation and Cooperative Guidance, IEEE International Conference on Electrical and Control Engineering (ICECE), Wuhan, China, 2010.

[9] Mingfeng Zhang; Hugh H. T. Liu, Formation flight of multiple fixed-wing unmanned aerial vehicles, IEEE American Control Conference, Washington, USA, 2013.

[10] Huan Liu; Xiangke Wang; Huayong Zhu, A novel backstepping method for the three-dimensional multi-UAVs formation control, IEEE International Conference on Mechatronics and Automation (ICMA), Beijing, China, 2015.

[11] Fang Liao, Xiangxu Dong, Feng Lin, Rodney Teo, Jian Liang Wang, Robust formation and reconfiguration control of multiple VTOL UAVs: Design and flight test, IEEE $22^{\text {nd }}$ Mediterranean Conference of Control and Automation (MED), Palermo, Italy, 2014.

[12] Ruibin Xue, Gaohua Cai, Formation Flight Control of Multi-UAV System with Communication Constraints, Journal of Aerospace Technology and Management, Vol. 8, 2016.

[13] Johnson Y and Dasgupta S., Robust Controller Design and Performance of Forward-velocity Dynamics of UAVs in Close Formation Flight, IEEE International Conference on Advances in Green Energy (ICAGE), Thiruvananthapuram, India, 2014.

[14] Fabio A. de Almeida, Tight Formation Flight with Feasible Model Predictive Control, AIAA Guidance, Navigation, and Control Conference, Kissimmee, Florida, 2015.

[15] Shixin Mao1, Wee Kiat Tan, and K. H. Low, Autonomous Formation Flight of Indoor UAVs Based on Model Predictive Control, AIAA Infotech @ Aerospace San Diego, California, USA, 2016.

[16] Haibin Duan, and Qinan Luo, Integrated Localization System for Autonomous Unmanned Aerial Vehicle Formation Flight, $12^{\text {th }}$ IEEE International Conference on Control \& Automation (ICCA), Kathmandu, Nepal, 2016.

[17] Sarper Kumbasar1 and Ozan Tekinalp, Fuzzy Logic Guidance of Formation Flight, IEEE International Conference on Unmanned Aircraft Systems, Denver, CO, USA, 2015.

[18] Ozan Tekinalp and Sarper Kumbasar, SDRE Based Guidance and Flight Control of Aircraft Formations, AIAA Guidance, Navigation, and Control Conference, Kissimmee, Florida, 2015.

[19] Stevens B.L. and F.L. Lewis, Aircraft Control and Simulation, John Wiley \& Sons, Inc. second edition, 2003.

[20] Rafael Socas, Sebastian Dormido and Raquel Dormido, Event-based control strategy for the guidance of the Aerosonde UAV, IEEE European Conference on Mobile Robots (ECMR), pages 1-6, Lincoln, UK, 2015. 
[21] B. Yun, B. M. Chen, K. Y. Lum and T. H. Lee, A leader-follower formation flight control scheme for UAV helicopters, IEEE International Conference on Automation and Logistics, Qingdao, China, pp. 39-44, 2008.

[22] Samarjit Kar, Sujit Das, and Pijush Kanti Ghosh, Applications of neuro fuzzy systems: A brief review and future outline, Elsevier Applied Soft Computing, Vol. 15, Pages 243-259, 2014.

[23] John Darvill, Alin Tisan, and Marcian Cirstea, An ANFIS-PI Based Boost Converter Control Scheme, $13^{\text {th }}$ IEEE International Conference on Industrial Informatics (INDIN), Cambridge, UK, 2015.

[24] Amr Sarhan, Shiyin Qin, Autonomous Intelligent Flight Control of Fixed-Wing UAV Based on Adaptive Neuro-Fuzzy Inference System, International Journal of Research in Engineering and Technology, Vol.5, 2016. 